\title{
Retos en pandemia para la equidad en acceso a formación inicial docente: estudio de caso en la Universidad Pedagógica Veracruzana
} Challenges during the pandemic regarding equity in access to initial teacher
training: case study at Universidad Pedagógica Veracruzana Desafios da pandemia para a equidade no acesso à formação inicial de professores: estudo de caso na Universidad Pedagógica Veracruzana

\author{
Oscar Valencia Aguilar \\ Universidad Pedagógica Veracruzana \\ Veracruz, México \\ ovalencia@msev.gob.mx \\ (D) ORCID: https://orcid.org/0000-0003-3029-2692
}

\author{
Recibido - Received - Recebido: 23 / 07 / 2021 Corregido - Revised - Revisado: 12 / 11 / 2021 Aceptado - Accepted - Aprovado: 25 / 11 / 2021 \\ DOI: https://doi.org/10.22458/ie.v24i36.3665 \\ URL: https://revistas.uned.ac.cr/index.php/innovaciones/article/view/3665
}

\begin{abstract}
Resumen: El encierro social derivado de la pandemia por la COVID-19, provocó a nivel mundial problemáticas en educación, en el ciclo escolar 2020-2021, ante esta nueva normalidad, se encontraron dilemas en las instituciones de educación superior, para fomentar la equidad en el acceso a la educación universitaria. El presente artículo tiene por objetivo describir las acciones de política pública educativa que se implementaron en la Universidad Pedagógica Veracruzana (UPV) -como estudio de caso-, para atender la equidad en el acceso a la formación inicial docente para el ciclo escolar 2020-2021. A partir de una metodología cuantitativa y con el uso de diversas fuentes de evidencia, se analizó una base de datos integrada por 2.260 registros, además de una investigación documental retrospectiva. Los resultados más relevantes del presente estudio se enfocan en dos elementos: el análisis pre decisional de una política pública educativa implementada para atender la promoción de equidad en el acceso a la universidad; es decir, se muestra el problema público, las posibles opciones para atenderse, la toma de decisiones por medio de una estrategia académica y su aplicación; además, describe a los aspirantes y los resultados de sus logros en el proceso. En conclusión, se identifica un caso exitoso promoviendo el logro de los aspirantes universitarios al motivar el uso de las Tecnologías de la Información y la Comunicación con una relevancia desde la perspectiva académica y social, la cual permite buscar otras rutas para la investigación educativa, con visión prospectiva en la equidad de la educación superior.
\end{abstract}

Palabras clave: acceso a la educación, enseñanza superior, estudiante universitario, formación profesional superior, política educacional.

\begin{abstract}
The social confinement derived from the COVID-19 pandemic caused worldwide problems in education during the 2020-2021 school year. In the face of this new normality, dilemmas were found in higher education institutions, to promote equity in access to higher education. The purpose of this article is to describe the educational public policy actions implemented at Universidad Pedagógica Veracruzana (UPV) -as a case study - to address equity in access to initial teacher training for the 2020-2021 school year. Based on a quantitative methodology and using various sources of evidence, a database of 2,260 records was analyzed, in addition to a retrospective documentary research. The most relevant results of this study focus on two elements: the predecisional analysis of an educational public policy implemented to address the promotion of equity in university access; in other words, it shows the public problem, the possible options to address it, the decision making process by means of an academic strategy and its application; it also describes the applicants and the results of their achievements in the process. In conclusion, a successful case is identified promoting the achievement of university applicants by motivating the use of Information and Communication Technologies with relevance from the academic and social perspective, which allows seeking other routes for educational research, with a prospective vision in the equity of higher education.
\end{abstract}

Keywords: Access to education, higher education, university students, higher professional training, educational policy. 
Resumo: O confinamento social derivado da pandemia da COVID-19 causou problemas mundiais na educação, no ano escolar de 2020-2021. Diante desta nova normalidade, foram encontrados dilemas nas instituições de ensino superior para promover a equidade no acesso à educação universitária. Este artigo visa descrever as ações de políticas públicas educacionais implementadas na Universidade Pedagógica Veracruzana (UPV) -mediante um estudo de caso- para tratar da equidade no acesso à formação inicial de professores para o ano escolar 2020-2021. Com base em uma metodologia quantitativa e utilizando várias fontes de evidência, foi analisado um banco de dados de 2.260 registros, além de pesquisas documentais retrospectivas. Os resultados mais relevantes deste estudo concentram-se em dois elementos: a análise pré-decisão de uma política pública educacional implementada para abordar a promoção da equidade no acesso à educação superior; ou seja, mostra o problema público, as opções possíveis para resolvê-lo, o processo de tomada de decisão através de uma estratégia acadêmica e sua aplicação; além disso, descreve os candidatos e os resultados de suas realizações no processo. Em conclusão, é identificado um caso de sucesso promovendo a realização de candidatos universitários motivando o uso de Tecnologias de Informação e Comunicação com relevância do ponto de vista acadêmico e social, o que permite procurar outros caminhos na pesquisa educacional, com uma visão prospectiva na equidade do ensino superior.

Palavras-chave: Acesso à educação, ensino superior, estudante universitário, formação profissional superior, política educacional

\section{INTRODUCCIÓN}

El acceso a la universidad es un tema por demás latente, en el 2019 se realizó una reforma educativa al Artículo Tercero Constitucional en México, la cual especifica que la obligatoriedad de la educación superior corresponde al Estado, mediante el ofrecimiento de oportunidades de acceso a todo aspirante (Ibarra, 2019).

Este proceso de gratuidad y garantía de acceso a la educación superior se identifica como elemento utópico, pero no imposible, técnicamente se requiere un arduo trabajo por parte del gobierno de México para atender la reforma educativa de 2019. Sin embargo, en este país se atendieron las recomendaciones internacionales para cuidar a la población de la pandemia derivada por la COVID-19. Esto implicó un aislamiento social que provocó una nueva normalidad, lo que derivó en diversos ajustes en el ámbito educativo: primero, una cuarentena a partir del 20 de marzo de 2020; en un segundo momento, el cierre del ciclo escolar 2019-2020 en estas condiciones y, en tercer lugar, el inicio del correspondiente a 20202021. Se cerraron los planteles escolares, pero no se dejó de atender la educación.

Por lo tanto, el presente estudio se justifica a partir de que no se encontraron elementos suficientes para identificar cuáles apartados de política pública educativa se implementaron en las instituciones formadoras de docentes, para ayudar a los alumnos a que continuaran con los estudios a nivel universitario, lo cual conlleva a una reflexión académica para su discusión posterior. Además, se alcanza una justificación técnica para tratar nuevos aportes al campo de la disciplina de las políticas públicas educativas al mostrar un análisis pre decisional. Independientemente de la inquietud del autor, con el fin de profundizar en los diversos enfoques teóricos y técnicos que permiten mostrar diferentes autores para esta temática del ingreso a los estudios superiores.

Lo anterior, permite dar cuenta de algunas características del estudio presentado en función de que las instituciones de educación superior (IES), en lo general no recibieron indicaciones precisas para atender el servicio que prestan, a diferencia de cómo se realizó en el nivel de educación básica en México, situación que implicó generar acciones de política pública educativa desde una perspectiva o visión creativa, para ello se toma en cuenta las múltiples limitantes encontradas por la nueva normalidad.

En años anteriores, para el tema del ingreso a la universidad, a los aspirantes a educación superior en México se les evaluaban la comprensión lectora, conocimientos específicos relacionados con la carrera, dominio en el idioma inglés como lengua extranjera, características personales, escolares y sociales, por 
medio de un examen diagnóstico llamado EXANI-II, que se presentaba de manera presencial (Centro Nacional de Evaluación para la Educación Superior, 2021).

Es importante mencionar que, esta investigación forma parte de un estudio de larga data inscrito al área de investigación de la UPV, dentro de la Línea de Generación y Aplicación del Conocimiento llamada Políticas, instituciones, sujetos y actores educativos, con la cual se abre un espacio de formación y análisis crítico en torno a tres de los principales aspectos y desde estos se constituye el campo de lo educativo, a saber: las políticas, las instituciones y los sujetos que las habitan; por tanto, estos tres aspectos, que desde aquí se plantean como nodales ya que vertebran al campo, se han de abordar siempre en relación complementaria, y no como aspectos separados entre sí.

Así, la indagación presentada se desprende de datos revelados en el estudio "Acciones de la UPV en la Pandemia por COVID-19", que busca interpretar las experiencias escolares de aprendientes de la UPV en esta temporalidad -para la Universidad Pedagógica Veracruzana se utiliza el término aprendiente a partir de la fundamentación teórica y curricular, así como en concordancia con la perspectiva constructivista de los diversos programas de estudio-, así como estar en correspondencia con el modelo pedagógico denominado Horizonte Educativo y se refiere a toda persona que aprende en el aprender durante toda su vida, en todo contexto. Además, los resultados iniciales han generado diversos reportes parciales de la investigación en torno a este fenómeno, tales como los estudios: tensiones caórdicas entre políticas públicas educativas y acciones institucionales (Valencia, 2021), análisis de las políticas públicas para el ingreso, las motivaciones de los aprendientes de la UPV, entre otros que están en proceso.

Ante este panorama, se formulan una serie de preguntas para identificar el problema que enfrentó una universidad pública estatal formadora de docentes: ¿cómo apoyar a los alumnos del nivel inmediato anterior a continuar con sus estudios universitarios ante la nueva normalidad?, ¿qué acciones de política pública educativa realizó la UPV para fomentar la equidad en el acceso a la educación superior en el ciclo escolar 2020-2021?, ¿quiénes son los aspirantes que participaron en el proceso de ingreso a una universidad pública estatal enfocada a la formación inicial docente?

Las preguntas anteriores muestran una problemática a manera de pregunta de investigación: ¿cuáles fueron las acciones de política pública educativa que desarrolló la UPV para fomentar la equidad en el acceso a la educación superior, ante la nueva normalidad para iniciar el ciclo escolar 2020-2021?

Como antecedentes, se menciona que la institución focalizada es una universidad pública estatal que tiene la función principal de "formación de profesionales de la educación, investigación en materia educativa y la difusión cultural de acuerdo a las necesidades del país" (Gaceta Oficial, 30 de septiembre de 1980). La Universidad Pedagógica Veracruzana (UPV) tiene establecidos sus objetivos en su decreto:

1. Formar recursos humanos del más alto nivel en educación básica; 2. Participar en la investigación y campos del conocimiento que coadyuven con la educación; 3. Reforzar la Educación mediante la formación de profesores especializados; 4. Participar en el desarrollo de la comunidad a través de programas que difundan el conocimiento científico y el arte; $y, 5$. Perseverar, acrecentar y difundir la cultura nacional y universal. (Gaceta Oficial, 30 de septiembre de 1980)

Esta institución de educación superior (IES) formadora de docentes atiende a 3.610 aprendientes en dos licenciaturas, una especialidad, seis diplomados, cuatro maestrías y un doctorado en quince Centros Regionales (CR) a lo largo y ancho del estado de Veracruz (Gobierno del Estado de Veracruz, 2018). Para atender las actividades escolares la UPV estos quince CR (equivalentes a sedes) se ubican en: Pánuco, Tantoyuca, Naranjos, Tuxpan, Papantla, Martínez de la Torre, Xalapa, Coatepec, Veracruz, Córdoba, Orizaba, Ciudad Mendoza, Cosamaloapan, San Andrés Tuxtla y Minatitlán (Universidad Pedagógica Veracruzana, 2020). 
Además, se destaca que en la UPV se cuenta con un modelo educativo denominado Horizonte Educativo (Velasco, Peredo, Fuentes, González, y López, 2016), el cual involucra a la mediación pedagógica que invita a "desarrollar el pensamiento divergente [...] esencial en el proceso de aprender en el aprender, lo que coadyuva a transitar de la ausencia de la emergencia de un comportamiento efectivo, permanente" (p. 139). Así, este horizonte está fundamentado en el aprender en el aprender y cuya tesis establece que para la formación inicial de los docentes se requiere incorporar elementos como la auto-organización, la autonomía, la creatividad, la comunicación, el lenguaje, la intersubjetividad (trabajo colaborativo), la ética, la interculturalidad, las Tecnologías de la Información y la Comunicación (pp. 91-117).

Para establecer un breve marco teórico, con respecto a la equidad en el acceso a la educación superior, se revisaron estudios de Lemaitre (2005), Espinoza, González y Latorre (2009), Rawls (2002), McCowan (2016), UNESCO (1998), Brighouse y Swift (2006), Silva (2014). Para las políticas públicas se retomó la perspectiva de Dye (2005), Valenti y Flores (2009), Aguilar (2004), Parsons (2007), Villarreal (2010) y para las políticas públicas educativas se retoma a Zorrilla \& Villa (2003).

Para argumentar el referente teórico de la equidad en el acceso a la educación superior, es importante retomar de la Organización de las Naciones Unidas para la Educación, la Ciencia y la Cultura (UNESCO, por sus siglas en inglés, 1998) el texto de La Educación superior en el siglo XXI, visión y acción: informe final, el cual expresa que "de conformidad con el párrafo 1 del Artículo 26 de la Declaración Universal de Derechos Humanos, el acceso a la educación superior ha de ser igual para todos, en función de los méritos respectivos" (UNESCO, 1998, p. 2); es decir, como política global existe una equidad de oportunidades, siempre y cuando se atiendan los méritos señalados.

Esto último difiere con lo encontrado en estudios de algunos países; pues en la actualidad no están disponibles los recursos para instalar el sistema educativo de educación superior que se requiere, por mencionar algunos ejemplos para integrar el presente estudio: Brasil, que tiene ingresos medios, con la educación superior con la antesala de una etapa de masificación y Kenia, estado de bajos ingresos con el nivel superior en expansión, pero restringido a una población baja (McCowan, 2016). Por otro lado, "la falta de recursos actuales no debiera limitarnos para pensar sobre una distribución justa" (McCowan, 2016, p. 16). El término de equidad en algunos textos se concibe como una inclinación hacia la "justicia", con un sentido opuesto a la igualdad; este significado se aproxima al de igualdad de oportunidades. Por lo tanto, los temas de equidad son caracterizados por el mismo nivel de debate que las encontradas para definir la justicia.

Para llegar a un entendimiento de la equidad, es considerable atender la diferenciación entre igualitarista y suficientista en torno a la justicia social, estos elementos fueron desarrollados de manera amplia en los textos de Brighouse y Swift (2006). No se puede dejar de contemplar los principios de Rawls (2002), teórico de la filosofía política de los años setenta, quien hizo un señalamiento muy claro para garantizar un estado de justicia social:

Cada persona tiene el mismo derecho irrevocable a un esquema plenamente adecuado de libertades básicas iguales que sea compatible con un esquema similar de libertades para todos. igualdad equitativa de oportunidades; $y$, en segundo lugar, las desigualdades deben redundar en un mayor beneficio de los miembros menos aventajados de la sociedad (Rawls, 2002, p. 73).

Con base en lo planteado por diversos autores, se retoma que para efectuar una aproximación hacia el marco conceptual de las políticas de educación superior si son equitativas o no, en el presente documento se aluden los conceptos de disponibilidad, accesibilidad y horizontalidad, como lo menciona McCowan (2016). La disponibilidad se comprende por el número de espacios disponibles en las IES, esto conlleva pensar en los docentes, equipamiento, por mencionar algunos elementos; en relación con la accesibilidad es importante visualizar los aranceles económicos, exámenes de ingreso, localización 
geográfica de las escuelas, por describir algunas que podrían generar problemas de estratificación; y, respecto a la horizontalidad que se expresa por una alta calidad y reconocimiento consistente en las escuelas (pp. 31-33).

Espinoza et al. (2009), emiten una definición del modelo de equidad que se expresa como "garantizar que todas las personas que tienen las mismas necesidades, capacidades y logros obtengan la misma cantidad de recursos financieros, sociales y culturales" (p. 99), además enfatizan cuatro dimensiones: acceso, permanencia, logro, resultados.

Por otro lado, el estudio de Lemaitre (2015) describe el concepto de equidad en la educación superior, para el caso chileno y lo menciona como extremadamente complejo. Expresa los elementos a considerar y coincide, en lo general, con los mencionados con McCowan, pero agrega los resultados y se refiere a que, entre más alto el nivel de estudios obtenidos, se vería reflejado en el nivel de ingresos económicos de las personas (pp. 77-78).

Desde otra perspectiva, Silva (2014), realiza un análisis de los diferentes instrumentos jurídicos que están vigentes en México para mostrar el sustento y comprender la equidad desde una visión normativa. Además, advierte que en las sociedades de América Latina se visualizan desigualdades y con diversas estratificaciones; por lo tanto, muestra que existen dos factores que no dependen de los alumnos y en su rendimiento participan: i) capital cultural (entendido como el ambiente con el que ha crecido y vive); y ii) calidad de la escuela (es el andamiaje académico en el que se desarrolló), esto ocasiona una distorsión en los procesos de selección (p. 26). Por ello, explica que la equidad en educación superior se entiende como:

[...] distribuir los recursos y procesos educativos de manera que los jóvenes provenientes de los estratos sociales más desfavorecidos tengan acceso a una educación de calidad y desarrollen procesos de aprendizaje significativos que les permitan beneficiarse de la misma para mejorar sus condiciones de vida (Silva, 2004, pp. 26-27).

Se destaca que esta autora menciona también tres grandes componentes en educación superior: acceso efectivo (opciones educativas relevantes), permanencia (procesos de aprendizajes significativos), resultados (beneficios de la escolaridad). Para efectos del presente documento se retoma la definición de esta última autora que coincide con los teóricos antes señalados y se declara que el análisis de los datos y los resultados se realizó a partir de la vertiente del acceso.

En ese mismo orden de ideas, la diversidad en la noción política pública resulta abundante y altamente significativa en contenido. Así, con la intención de contribuir a la explicación y comprensión del concepto de política pública, seguidamente se muestran algunas unidades de sentido -configuradas y otorgadas desde distintos locus de enunciación-, los cuales resultan útiles en virtud de que precisan rasgos de identidad de la política pública en tanto disciplina. Para Dye (2005) la política pública es todo lo que los gobiernos escogen hacer o no hacer. Son instrumentos del gobierno que conllevan elementos determinantes en su generación y su establecimiento: actores, procesos y formas organizativas. Su reto está en la implementación.

Desde otra perspectiva se identifica como una disciplina que "estudia los problemas públicos y decisiones a través de la utilización de enfoques multidisciplinarios y multi-metódicos con el propósito fundamental de resolver problemas que obstaculizan el bienestar público" (Valenti y Flores, 2009, p. 170). O bien, como lo señala Aguilar: El objetivo disciplinario de la Política Pública, las Policy Sciences/ Policy Analysist, desde su fundación académica en los años cincuenta, consistió en estudiar y racionalizar la policy-decision making, el proceso de diseño-decisión de las políticas para fines públicos (Aguilar, 2004, p. 24). 
Por lo tanto, política pública se entiende como: acción gubernamental que implica la elaboración de políticas compatibles con el marco constitucional y se sustancian con la participación intelectual y práctica de los ciudadanos, sin afectar las libertades y oportunidades de la población. Desde las Ciencias Políticas, el análisis de las políticas públicas tiene dos orientaciones: el estudio del proceso de las políticas y el estudio del conocimiento en el proceso de las políticas (Parsons, 2007 y Aguilar, 2004). Ambos tipos de análisis, aunque concomitantes, se distinguen básicamente en cuanto a que el primero focaliza en el proceso de políticas (policy process) para la resolución de problemas -problem solving (Aguilar, 2004, p. 35)- y el segundo en el proceso de toma de decisiones - policy-decision making-.

El análisis de las políticas públicas puede tener dos vertientes: el insumo de su creación -pre decisión, que implica la identificación del problema público, formulación de alternativas y toma de decisiones, entre otros- y la revisión de sus resultados -post decisión, como la aplicación, interpretación, evaluación, entre otros- (Parsons, 2007, pp. 53-59). A lo anterior, se destaca que en la literatura se encuentran diferentes modelos del ciclo de las políticas, la mayoría se identifican con un ciclo de cinco pasos en la que, a cada cual le corresponde un sistema de acción específico, actores y relaciones particulares, así como compromisos y estructuras sociales. Aquí vale indicar que, se retoma la dimensión de análisis de las políticas públicas, en este caso la sustantiva la cual "corresponde al proceso de formulación de políticas y toma de decisiones" (Villarreal, 2010, p. 266).

Para redondear este básico apartado teórico, por políticas públicas educativas se retoma la noción de Zorrilla y Villa (2003), quienes lo conciben de la siguiente manera:

[...] conjunto de orientaciones, lineamientos o criterios de carácter estratégico, es decir, destinados a facilitar el logro de determinadas finalidades en las que pueda sustentarse la relevancia, eficacia, eficiencia, impacto o equidad de las decisiones que se adopten y las acciones que se emprendan con el propósito de atender o cambiar los insumos, procesos y productos de un sistema educativo. (p. 32).

A partir de estas referencias, el objetivo general de este estudio es describir las prácticas de política pública educativa que se implementó en la UPV para atender la equidad en el acceso a la educación superior, ante la nueva normalidad para el ciclo escolar 2020-2021, con el fin de develar la dimensión sustantiva en el análisis de las políticas públicas educativas. Como objetivos específicos se declaran: a) identificar cuáles son las acciones de política pública educativa que permiten fomentar la equidad en el acceso a la educación superior en la UPV; b) mostrar los elementos para realizar un análisis pre decisional para apoyar a los alumnos a continuar con sus estudios universitarios ante la nueva normalidad; $y$, c) enunciar las características de los aspirantes que participaron en el proceso de ingreso a la UPV en el ciclo escolar 2020-2021.

\section{MATERIALES Y MÉTODOS}

El enfoque que se utilizó en esta investigación es cuantitativo, con un alcance descriptivo. El análisis de la información este espacio se divide en tres grandes aspectos: i) Las acciones de política pública educativa para fomentar el acceso a la educación superior, ii) El análisis predecisional de las políticas públicas educativas en la UPV; para los dos primeros fue necesario el uso de instrumentos cualitativos, como fue el análisis documental retrospectivo; y, iii) Los aspirantes que se incorporaron en el ciclo escolar 2020-2021.

Como instrumentos de recolección utilizados, en un primer momento se requirió el análisis documental retrospectivo, para identificar la aplicación de políticas públicas educativas para fomentar la promoción de la equidad -en la nueva normalidad- a la educación superior, desde una perspectiva pre decisional, con lo que se realizó una revisión del ciclo de las políticas públicas educativas en el periodo escolar 
2020-2021 y fueron desarrolladas e implementadas por la UPV, para fomentar el ingreso a la formación inicial docente.

En un segundo momento, el otro instrumento fue una base de datos con 2.260 registros que se integró derivado de la aplicación de la estrategia académica para mostrar el logro de los aspirantes a ingresar a la universidad, con este elemento de análisis se describen los referentes cuantitativos de los aspirantes, lo cual permite hacer un comparativo de los promedios obtenidos, así como la caracterización de estos.

Ahora bien, este es un estudio de caso en función de tres criterios: la indagación permite estudiar un tema determinado; se identifica un fenómeno contemporáneo en su entorno real, y; permite estudiar estos últimos desde múltiples perspectivas (Martínez, 2016). Para el primer criterio, se identifican las políticas públicas educativas, como acciones del gobierno de la UPV para el ingreso a una entidad de formación docente, el segundo se muestra en época de pandemia por la COVID-19 y el tercer elemento se identifica con la revisión documental retrospectiva y la base de datos integrada por el proceso. La validez es de construcción, por las técnicas de uso de diversas fuentes de información documental o evidencia en la fase de obtención de datos (Martínez, 2016, p. 178).

Cabe señalar que el presente documento sirve como elemento para generar investigación educativa, en términos de Reimers y McGinn (2000), "la utilización de investigación [educativa] no solo ayuda a los tomadores de decisiones a elegir cursos de acción sino a identificar cuáles son sus opciones" (p.10). No se pretende mencionar que es un análisis exhaustivo ni definitivo; sin embargo, para efectos del presente texto se acerca a una mirada de los primeros resultados.

\section{DISCUSIÓN DE RESULTADOS}

El hallazgo principal del estudio se identificó al encontrar que la UPV implementó un ciclo de política pública educativa, desde una perspectiva pre decisional, con el que generaron opciones para atender la equidad en el acceso a la educación superior, encontrando características específicas, de los aspirantes a una carrera que ofrece la formación inicial docente.

\section{Las acciones de política pública educativa para fomentar el acceso a la educación superior}

En términos de Aguilar (2014), el análisis predecional de las políticas públicas se destaca en la etapa de la formulación de estas, en el marco del policy process, en el cual se delinea la ruta de acción de la entidad gubernamental, en este caso la rectoría de la UPV y forma parte de una dimensión comunicativa.

A partir de este rubro, la UPV elaboró acciones para el ingreso a la Licenciatura en Educación Básica (LEB) en el ciclo escolar 2020-2021, a partir del uso intensivo de las Tecnologías de la Información y la Comunicación, encaminadas a identificar los perfiles de ingreso de los solicitantes a la carrera que ofrece esta IES. Es decir, la propuesta se fundamentó en conocer la calificación del nivel inmediato anterior, cuáles son los intereses para ser docente con la elaboración de un texto y la evaluación de tres rubros (razonamiento verbal, razonamiento lógico matemático y conocimientos generales), todo esto de manera asincrónica en sustitución de un examen presencial. 


\section{El análisis pre decisional de las políticas públicas educativas en la UPV para promover la equidad en el acceso a la educación superior}

En este apartado, es importante destacar que el análisis pre decisional implicó tres pasos del ciclo de políticas públicas (Villarreal, 2010): 1. Identificación de un problema público, 2. Formulación de soluciones; y 3. Toma de decisión. Los apartados de 4. Implementación y 5. Evaluación corresponden al análisis post decisional. Es decir, se identificaron las acciones de política pública educativa que fomentaron la equidad en el acceso a la educación superior en la UPV; además, se hallaron elementos para realizar un análisis pre decisional de manera inicial, lo cual fomentó que los aspirantes pudieran continuar con sus estudios universitarios frente a la nueva normalidad.

Para el paso 1. Identificación de un problema público, se expresa que uno de los inconvenientes por la pandemia es el encierro social, esta nueva normalidad obligó a todas las escuelas a cerrar el quehacer académico in situ y trasladar la atención académica a los medios electrónicos: video charlas (Zoom ${ }^{\oplus}$ Google Meet ${ }^{\oplus}$, Microsoft Skype ${ }^{\circledast}$, por mencionar algunos), uso de plataformas educativas (Google Classroom $^{\oplus}$, Edmodo ${ }^{\oplus}$, Microsoft Teams $^{\circledR}$, entre otros). Esto para atender las clases por medios digitales.

En pasados ciclos escolares, el ingreso a la UPV era por medio de exámenes. Estas evaluaciones medían el desempeño de los aspirantes para obtener un lugar dentro de la Universidad, que eran espacios por demás limitados. Con la crisis del encierro social por la COVID-19, algunas IES tuvieron complicaciones por la aplicación del referido examen en el ciclo escolar 2020-2021, como ejemplo, algunas escuelas en México como la Universidad Autónoma de Sinaloa (UAS) y la Universidad de Guadalajara (UdeG), aceptaron alumnos sin concurso de ingreso. La Universidad Autónoma de Coahuila realizó un examen en línea, por medio de una empresa privada que ofreció los resultados encriptados. Por su parte, la Universidad Nacional Autónoma de México (UNAM) postergó en dos ocasiones su examen de ingreso por la pandemia (Sánchez, 2020).

Este último dato, permitió identificar que algunas IES en este país aún cuentan con problemas de logística para determinar cuáles aspirantes pueden ser aceptados dentro de sus aulas, para cursar los diferentes programas de educación superior que ofrecen. Para la UPV este inconveniente es aún mayor por diferentes circunstancias: a) no cuenta con una infraestructura física propia (por su fundación y visión social de actualización al magisterio); b) la plataforma educativa de su licenciatura está elaborada en Moodle (ver https://upvv.clavijero.edu.mx/login/) pero está instalada en un servidor de otra institución; $y, c)$ en procesos anteriores la única forma de ingresar era por examen aplicado por el Centro Nacional de Evaluación para la Educación Superior (CENEVAL), a través del EXANI-II.

Por su parte, el Gobierno del Estado de Veracruz implementó, por medio de la Subsecretaría de Educación Media Superior y Superior el Programa Emergente de Oferta Educativa (PEOE-2020), el cual integró en una sola plataforma a todos los subsistemas de nivel medio superior y superior, estatales, federales, públicos y particulares, de la entidad veracruzana para orientar a los aspirantes a ingresar a esos niveles educativos (Secretaría de Educación de Veracruz, 2020), con esta acción de política pública esa entidad gubernamental buscó promover el acceso al servicio educativo y así abatir el abandono escolar en esos niveles educativos. La UPV participó con 850 espacios en el PEOE, como se muestra en la Tabla 1. 
TABLA 1

Distribución de lugares disponibles para el ingreso a la Licenciatura en Educación Básica en el ciclo escolar 2020-2021

\begin{tabular}{lcc}
\multicolumn{1}{c}{ Centro Regional } & Disponibilidad de lugares & $\begin{array}{c}\text { Distribución porcentual } \\
\text { (\%) }\end{array}$ \\
Pánuco & 50 & 5,9 \\
Tantoyuca & 50 & 5,9 \\
Naranjos & 50 & 5,9 \\
Tuxpan & 50 & 5,9 \\
Papantla & 70 & 8,2 \\
Martínez de la Torre & 50 & 5,9 \\
Xalapa & 75 & 8,8 \\
Veracruz & 60 & 7,0 \\
Córdoba & 90 & 10,6 \\
Orizaba & 60 & 7,0 \\
Ciudad Mendoza & 75 & 8,8 \\
Cosamaloapan & 50 & 5,9 \\
San Andrés Tuxtla & 60 & 7,1 \\
Minatitlán & 60 & 7,1 \\
Total & 850 & \\
\hline & Fuente: convocatoria de Ingreso a la LEB 2020-2021.
\end{tabular}

En el paso 2. Formulación de soluciones, se llevaron a cabo diversos análisis para afrontar este inconveniente que se generó como problema público de la UPV, uno de ellos fue realizar el examen del CENEVAL, pero esto significaría el contacto físico y aglomeraciones en espacios físicos, otro fue valoración de promedio o generar un producto específico para la evaluación de los aprendientes y para ello se toman en cuenta diversos factores como: identificar los perfiles de los aspirantes, crear un producto escrito, promedio del nivel inmediato anterior y otras evaluaciones; todo esto a distancia, con ello se promueve la inclusión al ofrecer opciones de ingreso. Los datos mencionados en este apartado corresponden a documentos internos de trabajo con los que se mostró a los docentes que participaron en el proceso de evaluación la manera en cómo se desarrolló esta estrategia (Universidad Pedagógica Veracruzana, 2020a).

Con respecto al paso 3. Toma de decisión, implicó seleccionar la elaboración de un proceso de diagnóstico alternativo, por medio de un Taller multimodal que se concibió como un espacio dialógico asincrónico para identificar los perfiles de los aspirantes a la Licenciatura en Educación Básica (LEB) de la UPV, por medio del desarrollo de un producto integrador, el cual sirvió de referente en el ingreso que la UPV ofrece, específicamente el programa de estudios de la LEB.

Este taller tuvo como objetivo principal:

Construir un producto integrador que evidencie el empleo, de manera autónoma, de herramientas básicas para el desarrollo de procesos de autoexploración, reflexión y articulación de saberes en situaciones relacionadas con el ejercicio profesional docente, hacia la emisión de un diagnóstico generador de insumos académicos suficientes, que posibiliten la selección de los candidatos a ingresar al programa de Licenciatura en Educación Básica de la UPV (Universidad Pedagógica Veracruzana, 2020b). 
Esta estrategia se construyó para atender a una potencial población de 2.000 aspirantes y se contempló de la siguiente manera:

- A partir de Modelo Pedagógico de la UPV, se fomentó la pedagogía de la Auto-organización, la cual permitió que cada aspirante fuera responsable de su propio aprendizaje.

- El taller se programó a partir de cinco actividades (exploración de la dimensión personal, exploración de la dimensión interpersonal, exploración de la dimensión social, exploración de la dimensión axiológica, reflexión sobre la complejidad de la práctica docente) y se concretó con la elaboración de un documento final a partir de la construcción de un ensayo argumentativo.

- Los criterios de evaluación y acreditación fueron los siguientes:

- Aprobación del taller con una ponderación máxima de 60\%.

- Promedio obtenido del bachillerato con 10\% como máximo.

- Otras evaluaciones con 30\% como máximo (instrumento de valoración de habilidades, el cual consta de 60 reactivos que exploraron las habilidades de razonamiento verbal, razonamiento lógico-matemático y conocimientos generales).

- Se aplicó en dos vertientes, ambas asíncronas; sin embargo, la UPV las denominó de la siguiente manera:

- Modalidad en línea, se desarrolló a través de la plataforma Moodle, a la cual accesaron los aspirantes que la solicitaron.

- Modalidad a distancia, en este caso, los aspirantes ingresan al repositorio en donde encontrarán los recursos materiales para descargarlos y llevarlos a su casa a fin de analizarlos, desarrollar las actividades y construir el producto final, el cual debieron enviar a través del espacio del repositorio, esto por medio de correo electrónico en modalidad asincrónica.

- El producto del taller (ensayo argumentativo) se revisó por un evaluador que, a partir de los criterios de evaluación del ensayo académico, emitió una opinión neutral, pero sobre todo basada en el rigor académico (Universidad Pedagógica Veracruzana, 2020c).

\section{Los aspirantes que se incorporaron en el ciclo escolar 2020-2021}

En este proceso de ingreso a la LEB de la UPV en la nueva normalidad se incorporaron en un pre-registro de 2.600 personas; después de cumplir con los elementos de la convocatoria se integró una base de datos para la evaluación de 2.260 participantes. Esta base de datos sirvió para el análisis cuantitativo y se construyó en tres momentos: a) al registrarse los aspirantes capturaron su promedio del bachillerato (esta información fue validada en un segundo momento por el área responsable de control escolar de la UPV); b) cuando los evaluadores revisaron el ensayo argumentativo; $y, c)$ al finalizar el proceso, al presentar sus otras evaluaciones: pensamiento matemático, pensamiento analítico y habilidades comunicativas. Con estos puntos se enunciaron las características de los solicitantes que participaron en el proceso de ingreso a la UPV en el ciclo escolar 2020-2021.

Para atender esta acción se integraron 100 grupos con el mismo número de académicos que realizaron la función de evaluadores del ensayo argumentativo. Para cuidar el proceso se buscaron a docentes, en su mayoría externos a la UPV, según los documentos internos de la UPV analizados para esta indagación.

Los primeros datos mostraron que los CR más solicitados fueron Minatitlán, Córdoba, Orizaba y Xalapa; por otro lado, los CR con menor solicitud fueron: Martínez de la Torre, Naranjos, Pánuco y Tantoyuca. La distribución porcentual se muestra en la figura 1. 
Figura 1. Distribución porcentual de aspirantes a ingreso a la LEB de la UPV en el ciclo escolar 2020-2021

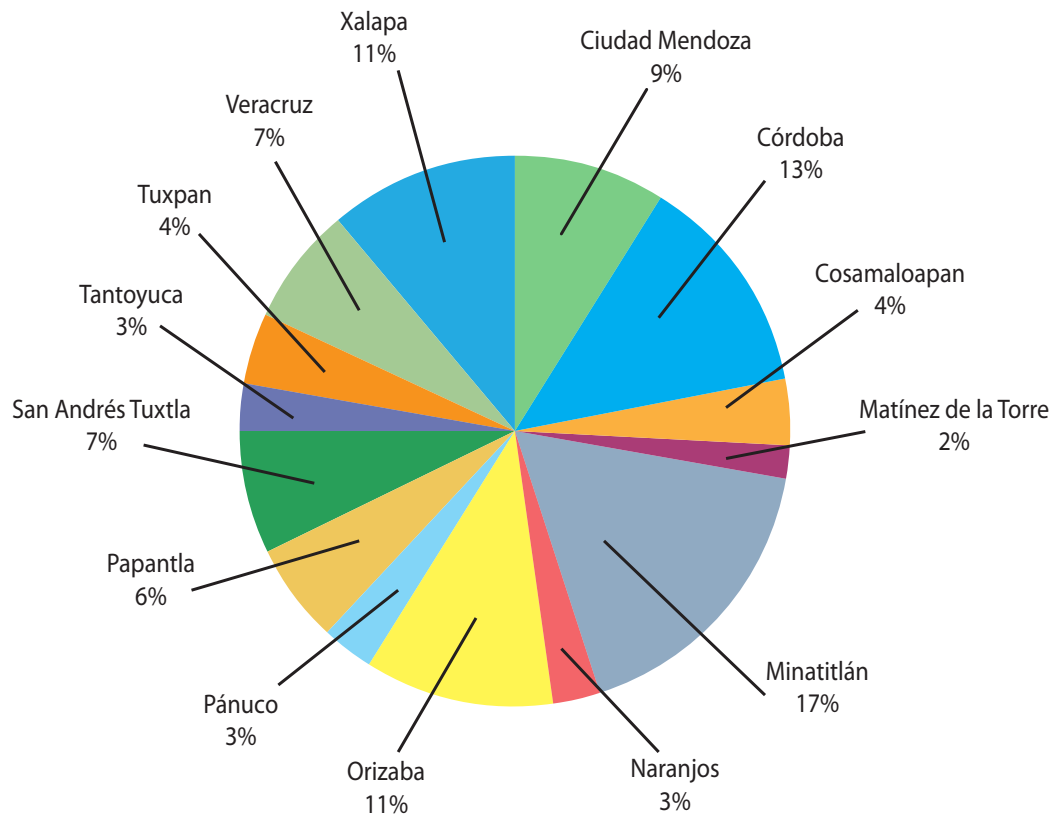

Fuente: elaboración propia a partir de la Base de datos de los aspirantes a ingreso a la LEB de la UPV en el ciclo escolar 2020-2021.

La modalidad que los aspirantes seleccionaron fueron diferenciadas, 58\% (1.308) utilizaron la plataforma de Moodle y $42 \%$ (952 aspirantes se declinaron por el uso de correo electrónico). Destaca el caso del CR Minatitlán en el que la mayoría de los solicitantes usaron la modalidad de correos electrónicos comparativamente con los CR de Papantla y San Andrés Túxtla que, en gran número, utilizaron la modalidad de la plataforma Moodle. Esto se muestra en la figura 2.

Figura 2. Distribución de la selección de los aspirantes para trabajar en los medios asincrónicos:

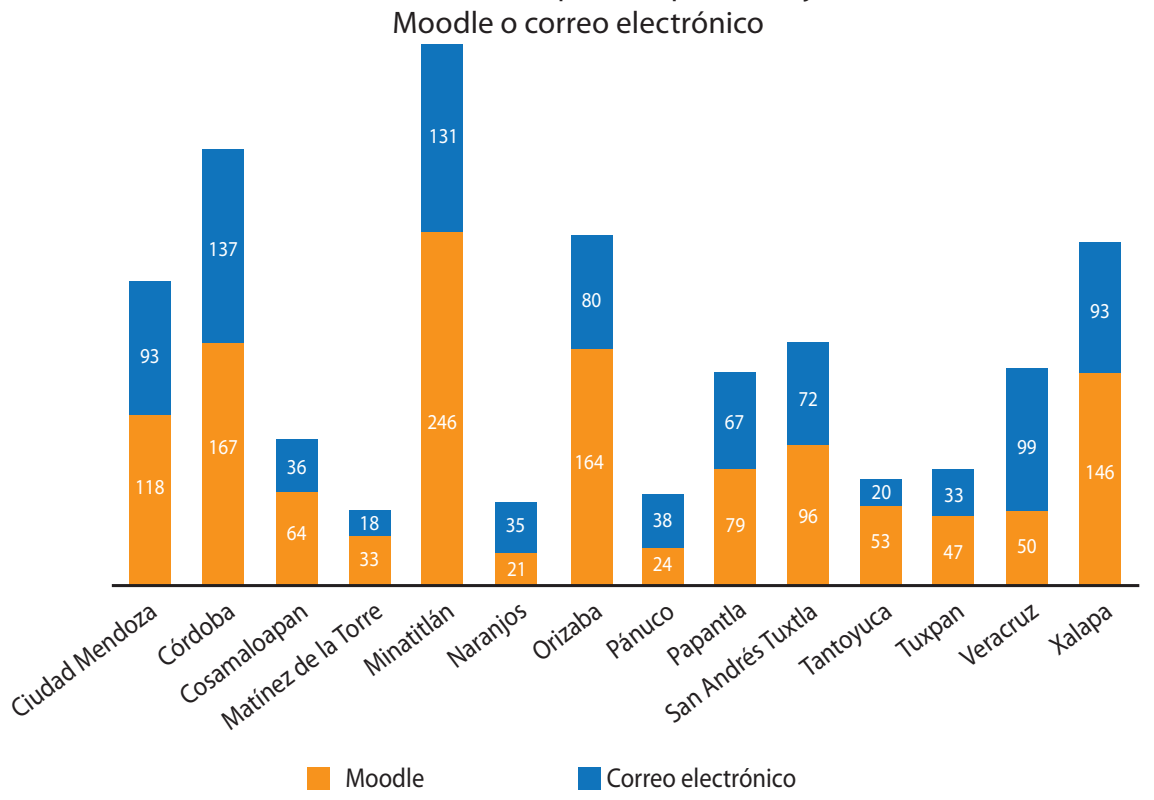

Fuente: elaboración propia a partir de la Base de datos de los aspirantes a ingreso a la LEB de la UPV en el ciclo escolar 2020-2021. 
Los resultados de la estrategia de ingreso derivaron en el análisis comparativo de los promedios por centro regional, por ejemplo, los promedios más altos con respecto al ensayo se reflejaron en el CR Martínez de la Torre con un promedio de 40,4 puntos de 60 y se complementa con 8,2 de promedio de calificaciones de egreso de bachillerato. Los promedios de resultados de bachillerato más altos son de los CR Martínez de la Torre, Veracruz y Pánuco con 8,2 (el máximo fue de 10). En relación con los resultados más altos del instrumento de valoración en pensamiento Matemático es el CR de Veracruz con 7,4, este mismo CR tiene el promedio más alto de pensamiento analítico, al igual que el promedio de habilidades cognitivas con 7,9; el total del curso, tiene el promedio más alto en el CR de Tantoyuca con 65,3. Estos resultados se identifican en la Tabla 2.

TABLA 2

Promedios de resultados obtenidos por los aspirantes a ingreso a la LEB de la UPV en el ciclo escolar 2020-2021

\begin{tabular}{|c|c|c|c|c|c|c|}
\hline \multirow{2}{*}{ Centro Regional } & \multicolumn{6}{|c|}{ Promedios } \\
\hline & Ensayo & Bachillerato & $\begin{array}{l}\text { 1. Pensamiento } \\
\text { Matemático }\end{array}$ & $\begin{array}{c}\text { 2. Pensamiento } \\
\text { Analítico }\end{array}$ & $\begin{array}{l}\text { 3. Habilidades } \\
\text { Comunicativas }\end{array}$ & del curso \\
\hline Ciudad Mendoza & 29,7 & 8,0 & 6,5 & 6,6 & 7,3 & 49,9 \\
\hline Córdoba & 32,1 & 8,0 & 6,9 & 6,5 & 7,3 & 56,2 \\
\hline Cosamaloapan & 35,2 & 8,0 & 6,5 & 6,2 & 7,3 & 60,3 \\
\hline Martínez de la Torre & 40,4 & 8,2 & 6,7 & 6,3 & 7,3 & 65,1 \\
\hline Minatitlán & 32,6 & 8,1 & 6,7 & 6,5 & 7,3 & 56,6 \\
\hline Naranjos & 29,5 & 7,7 & 6,5 & 6,6 & 7,2 & 57,1 \\
\hline Orizaba & 33,4 & 8,0 & 6,7 & 6,6 & 7,1 & 58,0 \\
\hline Pánuco & 29,5 & 8,2 & 6,7 & 6,3 & 7,1 & 53,3 \\
\hline Papantla & 31,6 & 7,9 & 6,7 & 6,5 & 7,5 & 53,0 \\
\hline San Andrés Tuxtla & 34,8 & 8,1 & 6,5 & 6,5 & 7,4 & 60,3 \\
\hline Tantoyuca & 39,8 & 7,9 & 6,2 & 6,6 & 7,3 & 65,3 \\
\hline Tuxpan & 34,6 & 8,1 & 6,4 & 6,7 & 7,3 & 60,3 \\
\hline Veracruz & 31,7 & 8,2 & 7,4 & 7,1 & 7,9 & 60,2 \\
\hline Xalapa & 33,9 & 7,9 & 7,2 & 7,0 & 7,6 & 59,3 \\
\hline Total general: & 33,0 & 8,0 & 6,7 & 6,6 & 7,3 & 57,3 \\
\hline
\end{tabular}

Fuente: elaboración propia a partir de la Base de datos de los aspirantes a ingreso a la LEB de la UPV en el ciclo escolar 2020-2021.

Otra forma de visualizar los resultados, es con un histograma de los promedios obtenidos en la evaluación final, este incorpora los resultados del ensayo, el promedio obtenido por los aspirantes en el bachillerato, así como los resultados del instrumento de valoración: 1. Pensamiento Matemático, 2. Pensamiento Analítico, 3. Habilidades Comunicativas. En ese sentido, se aprecia que los puntajes de 60 a 84 son los que más elementos tienen y se considera que la media es de 8,1, así como la mediana de 60,89 y muestra una asimetría hacia la izquierda, como se aprecia en la figura 3. 
Figura 3. Histograma de los resultados de los promedios obtenidos por los aspirantes a la LEB.

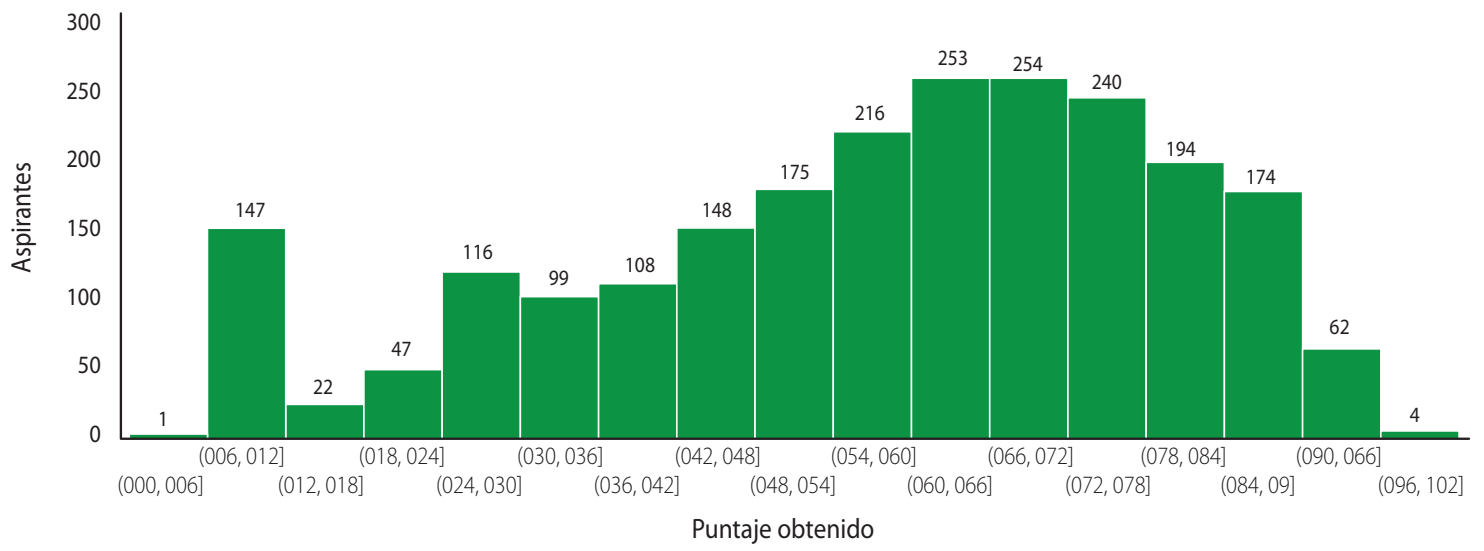

Fuente: elaboración propia a partir de la Base de datos de los aspirantes a ingreso a la LEB de la UPV en el ciclo escolar 2020-2021.

Como referente adicional, se elaboraron gráficos de Pareto de los resultados obtenidos por los aspirantes en las evaluaciones diagnósticas, los resultados en los promedios más altos son los siguientes: para el Pensamiento Matemático 403 sustentantes reflejaron un promedio de 7,30 a 8,03; Pensamiento Analítico 498 aspirantes obtuvieron 6,90 a 7,59 puntos en promedio; $y$, sobre las Habilidades Comunicativas 520 personas tuvieron un puntaje de 8,36 a 9,12 de promedio en este rubro. Estos referentes se muestran en la Figura 4.

Solo para señalar que la UPV determinó que el puntaje mínimo de ingreso fuera de 78,78 de promedio y el puntaje más alto fue de 91,75 . Estos primeros resultados cuantitativos permiten dar cuenta de la descripción de forma inicial de los aspirantes a estudiar en la LEB que oferta la UPV y, por motivos de la nueva normalidad, ingresaron por medio del Taller multimodal.

Figura 4. Representación de gráficos de Pareto de los resultados del instrumento de valoración: a. Pensamiento Matemático, b. Pensamiento Analítico, c. Habilidades Comunicativas.
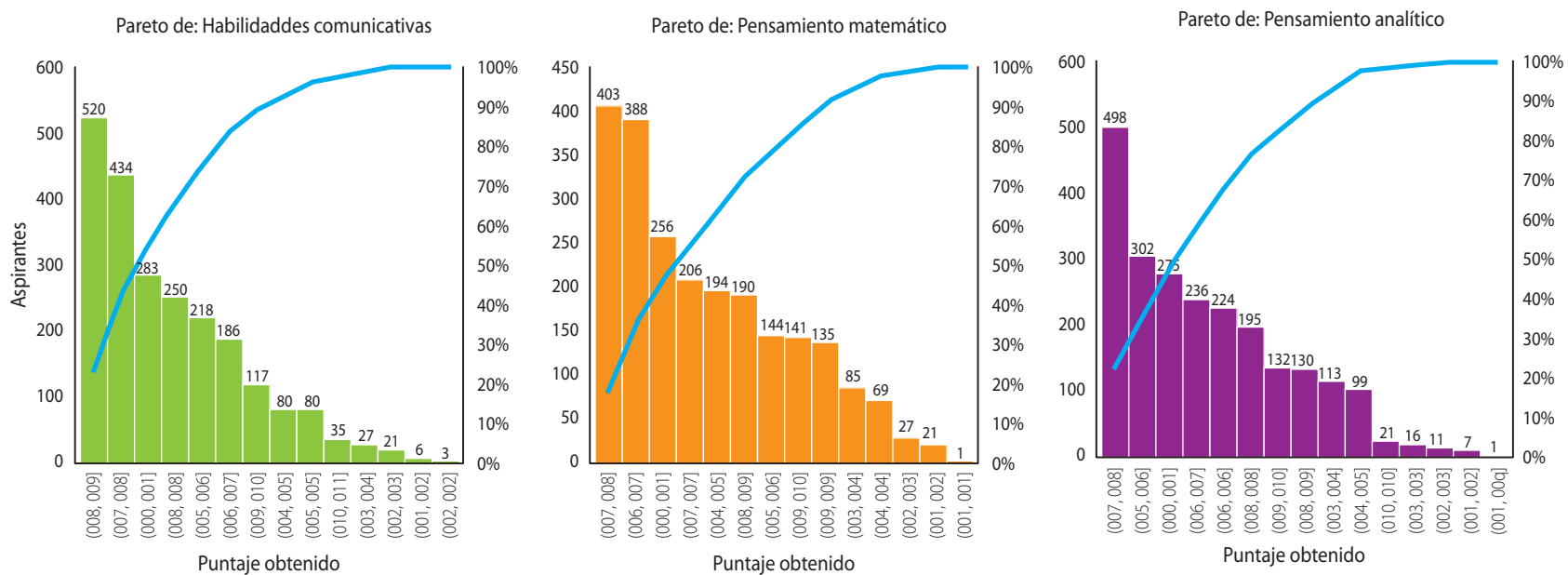

Fuente: elaboración propia a partir de la Base de datos de los aspirantes a ingreso a la LEB de la UPV en el ciclo escolar 2020-2021. 


\section{SÍNTESIS Y REFLEXIONES FINALES}

La metodología cuantitativa permitió obtener fuentes de información variada, la cual mostró un ejercicio de política pública educativa, desde el análisis pre decisional, por medio de la descripción de las acciones en la toma de decisiones de la UPV para promover la equidad en el acceso a la educación superior, esto con la revisión de documentos internos de la Universidad. Es importante señalar que la conformación de una política pública educativa implica elaborar un ciclo para llevar a cabo desde su análisis hasta su evaluación. Es este texto, solo se revisó al análisis pre decisional y se alcanza a verificar un elemento previo del post decisional.

Se identifica que se postularon $13 \%$ más de lo programado, en términos reales, esta estrategia se generó para atender a una población objetivo: interesados en ingresar a la LEB; sin embargo, este número programado se rebasó, pero no así las acciones de la UPV.

Por otro lado, se refleja un análisis inicial de los aspirantes a la LEB y sus logros, por medio de la base de datos integrada para este estudio. Dentro de las conclusiones que se muestran es que $11 \%$ de los lugares disponibles son para el CR de Córdoba; sin embargo, en la solicitud de los aspirantes, existe mayor demanda en el CR de Minatitlán, luego Córdoba y en el mismo porcentaje Orizaba y Xalapa, este dato seguramente servirá para poder mejorar la estrategia, si es que se decide continuar por la misma ruta.

Una de las características más importantes de los aspirantes es que el nivel académico que muestran con el promedio del bachillerato oscila en 8,0 . En lo general, se demuestra que el promedio del curso obtenido por los aspirantes es de 6,0, esto incluyendo el producto del taller, el promedio escolar y las otras evaluaciones. El dato aislado tal vez no significa mucho, pero da cuenta de cómo egresan los estudiantes del bachillerato; es decir, expresa el andamiaje cultural en los que se ha desarrollado previo al ingreso a la Universidad y se refleja en los análisis de Pareto elaborados para este texto.

Estos resultados son el esfuerzo y la dedicación de esta Universidad (personal directivo, operativo y académico) por dar continuidad al proceso de la formación inicial docente y difiere del estudio de Alcántara, en el cual se expresa que "instituciones universitarias han cerrado e intentado adoptar el aprendizaje en línea, muy pocas están bien preparadas para hacer este cambio de manera rápida y abrupta" (Alcántara, 2020, p. 76). En este caso, la UPV no ha causado confusión; por lo contrario, ha utilizado el Horizonte Educativo (Velasco et al., 2016) para generar elementos de auto-organización, lo cual demuestra un contraste con lo que se encontró en el estudio de referencia.

Además, otro punto referente es el estudio de Alcántara (2020), quien explica que -en diversos países-, algunas instituciones de educación superior que no han aceptado transitar a esta modalidad: caso de Argentina donde facultades pospusieron las clases y reorganizaron el calendario académico, bajo el argumento de la calidad en la educación, solo se alcanza con las clases presenciales. Otros ejemplos, en el mismo estudio, con tonos diferenciados en Zimbabwe, Malasia, Túnez, Chile y Reino Unido. Es decir, con esta emergencia sanitaria, se permite observar que la equidad en la educación superior es un gran desafío para grupos vulnerables (Alcántara, 2020, pp. 77-80).

Por otra parte, como plantea Umaña-Mata "uso de virtualidad no es sinónimo de educación a distancia" (2020, p.48), al incorporar el uso de las TIC en la educación para favorecer el aprendizaje en línea como opción para continuar con las actividades académicas; pero, en el presente estudio reveló que los aspirantes a formarse como docentes en la UPV están convencidas del uso de las tecnologías en esta época de crisis, para continuar con sus estudios por medio de un cambio cognitivo y toman esta opción como un modelo educativo tan válido como la que se utiliza en modalidad presencial, a partir de la filosofía del Horizonte Educativo (Velasco et al., 2016) que se ha venido aplicando en los últimos años, esta referencia se identifica al revisar el número de casos que aspiraron a ingresar a la LEB en la UPV. 
Si bien es cierto, existen elementos por atender de mejor manera, se destaca que se realizaron acciones precisas y pertinentes a partir de un análisis del ciclo de las políticas públicas desde la perspectiva pre decisional. No es una revisión exhaustiva, pero identifica elementos básicos para generar otros rubros de estudios. Tampoco se infiere de reporte institucional, dista mucho de este aspecto. Este estudio se enmarca desde la perspectiva de la policy-decision making; pues se realizó una toma de decisiones informada, sobre todo salvaguardando en todo momento la seguridad de los aspirantes a ingresar a LEB, así como de los diversos actores que participan en el proceso de ingreso: personal directivo, docente, técnico y autoridades de la institución.

En la revisión del análisis pre decisional se requieren otros elementos para generar una recomendación más detallada, por ejemplo: no se señala en este texto la apreciación de cómo vivieron el proceso los actores que evaluaron los ensayos y calificaron el $60 \%$ del valor total de ingreso a la LEB. Esta muestra podría explicar la otra parte del proceso, su mirada estuvo enfocada en los aciertos o errores que tuvieron los aspirantes.

Este estudio, como insumo para futuras investigaciones en el campo educativo, también muestra un escaparate para identificar otro análisis relacionado con la perspectiva post decisional de las políticas públicas educativas, sobre todo contribuir a una evaluación del proceso de implementación a partir de la toma de decisiones y la resolución de los problemas que se identificaron. Esto podría aportar recomendaciones desde las retroalimentaciones con miras a una mejora de los procesos y procedimientos, en función de que la pandemia aún no está controlada en México.

Cabe señalar que con los datos revisados se podría generar un análisis para mostrar algunas carencias de los egresados del bachillerato y focalizar cómo mejorar los procesos de enseñanza aprendizaje; es decir, que el trayecto formativo no sea una limitación para el ingreso a la Universidad, no solo como aspirantes a una IES ya sea formadora de docentes o de otro tipo, la referencia es para las diversas opciones de la Universidad. Desde el referente teórico, se podría atender que la calidad de la escuela, entendida como el andamiaje académico en que se desarrolló el aspirante a ingresar a la Universidad no sea un impedimento en los exámenes o los procesos de selección que determina como política global la UNESCO, al mencionar que la educación superior ha de ser igual para todos, en función de los méritos respectivos.

En ese sentido y como aportación, la mejora de la equidad en el acceso a la educación superior, requiere de una mirada prospectiva, para ello es fundamental retomar a Miklos y Tello (2015), quienes la describen como "preparar el camino, adaptándolo como objetivo (deseable y posible), es decir, guía las acciones presentes y el campo de lo posible del mañana". Además, encamina a los objetivos a) construir escenarios o imágenes; b) aportar elementos estratégicos; c) impulsar la planeación fundamentada en visión del futuro; y d) aportar una guía conceptual conductora del estudio.

Para Baena (2015) la previsión se muestra como una espiral y la incertidumbre se refleja como un abanico por las varias direcciones que conforma, esto a partir de los estudios de Deplhi (estudios de previsión de futuro). Para ella, la Planeación Prospectiva Estratégica es saber hacia dónde se tiene que llegar, en esta acción se determina el futuro deseado creativamente y libre de restricciones, se considera al pasado y el presente en un segundo momento. Una vez que se visualiza la imagen del futuro deseado se exploran las visiones factibles para seleccionar el elemento más satisfactorio.

Con estos últimos elementos de prospectiva implica re-pensar o re-configurar a la UPV, con el fin de retomar el Horizonte Educativo (Velasco et al., 2016) para generar una prospectiva institucional que permita atender lo cotidiano, entendido como lo normalizado, con todos los elementos de política pública educativa; así como atender los tiempos de crisis, que implican la nueva normalidad y se puedan atender en línea o distancia a partir de la intersubjetividad. 


\section{REFERENCIAS}

AlCÁntARA, A. (2020). Educación superior y COVID-19: una perspectiva comparada. En H. Casanova Cardiel (Coord.), Educación y pandemia: una visión académica (pp. 75-82). Ciudad de México: Universidad Nacional Autónoma de México, Instituto de Investigaciones sobre la Universidad y la Educación.

Aguilar, L. F. (2004). Recepción y desarrollo de la disciplina de Política Pública en México. Un estudio introductorio. Sociológica, 19(54),15-37. ISSN: 0187-0173. Recuperado de: https://www.redalyc.org/ articulo.oa?id=3050/305026633001

Aguilar, L. (2014). La hechura de las Políticas. México: Miguel Ángel Porrúa.

BAENA, G. (2015). Planeación prospectiva estratégica. Teorías, metodologías y buenas prácticas en América Latina. México: Universidad Nacional Autónoma de México.

Brighouse, H., \& SwIFT, A. (2006). Equality, Priority, and Positional Goods. Ethics, 116(3): 471-497. https://doi.org/10.1086/500524

Centro Nacional de Evaluación para la Educación Superior. (2021). Nivel Superior EXani II - Ceneval. (2020, octubre 28). Obtenido de: https://ceneval.edu.mx/examenes-ingreso-exani_ii/

Dye, R. (2005). Understanding Public Policy. (12th ed.), New Jersey, EE.UU.: Prentice Hall.

Espinoza, O., GonzÁlez, L. y LATORRE, C. (2009). Un modelo de equidad para la educación superior: Análisis de su aplicación al caso chileno. Revista de la educación superior, 38(150):97-111. Obtenido de: http://www.scielo.org.mx/scielo.php?script=sci_arttext\&pid=S0185-27602009000200006\&lng= es\&tlng=es.

Gaceta Oficial del Estado. (30 de septiembre de 1980). Decreto. Creación de la Universidad Pedagógica Veracruzana. Obtenido de: https://www.sev.gob.mx/upv/2011/11/15/decretos/

Gobierno del Estado de Veracruz (2018). La sociedad de Veracruz en cifras: Segundo Informe de Gobierno 2017-2018. Obtenido de: http://repositorio.veracruz.gob.mx/wp-content/uploads/sites/4/files/ transp/informes_2016_2018/segundo_informe/Tomo4.pdf

IbarRA, A. (19 de marzo de 2019). Reforma Educativa 2019: los cambios. Educación Futura. Obtenido de https://www.educacionfutura.org/reforma-educativa-2019-los-cambios/

Lemaitre, M.J. (2005). Equidad en la educación superior: un concepto complejo. REICE. Revista Iberoamericana sobre Calidad, Eficacia y Cambio en Educación, 3(2),70-79. Obtenido de: https://www.redalyc.org/articulo.oa?id=551/55103206

Martínez C., P. C. (2006). El método de estudio de caso: estrategia metodológica de la investigación científica. Pensamiento \& Gestión, (20):165-193. ISSN: 1657-6276. Obtenido de: https://www.redalyc.org/articulo.oa?id=64602005

McCowAN , T. (2016). Un marco conceptual sobre la equidad en el acceso a la educación superior. En Zúñiga C., Redondo J., López M., Santa Cruz E. (Ed.) Equidad en la educación superior: Desafíos y proyeccio-

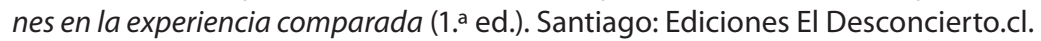

Miklos, R. T. y Tello, M. E. (2015). Planeación prospectiva: una estrategia para el diseño del futuro. México: LIMUSA S.A. de C.V.

PARsons, W. (2007). Políticas públicas: una introducción a la teoría y la práctica del análisis de políticas públicas. México: Facultad Latinoamericana de Ciencias Sociales.

RAwLS, J. (2002). La justicia como equidad. Barcelona: Paidós.

Reimers, F., y McGinn, N. (2000). Diálogo informado: El uso de la investigación para conformar la política educativa. EE. UU.: Centro de Estudios Educativos - Asociación de Universidades Confiadas a la Compañía de Jesús en América Latina. 
SÁNCHEZ,J.(2020). Universidades aceptan ajóvenes sin aplicarexamen de admisión. La Jornada. Obtenido de: https://www.jornada.com.mx/2020/07/19/politica/007n1pol

Secretaria de Educación de Veracruz (2020). Programa Emergente de Oferta Educativa. Obtenido de: https://semsys.sev.gob.mx/PEOE/PE.html

SILVA, M. (2014). Equidad en la educación superior mexicana: el reto persistente. Universidades, (59):2335. ISSN: 0041-8935. Obtenido de: https://www.redalyc.org/articulo.oa?id=373/37332547004

Universidad Pedagógica Veracruzana. (2020). Directorio de Centros Regionales. https://www.sev.gob.mx/ upv/directorio-de-centros-regionales-de-estudio/

Universidad Pedagógica Veracruzana. (2020a). Presentación "Exploración de herramientas básicas para el ingreso a la formación docente". Documento inédito de trabajo.

Universidad Pedagógica Veracruzana. (2020b). Taller de exploración de herramientas básicas para el ingreso a la formación docente. Documento inédito de trabajo.

Universidad Pedagógica Veracruzana. (2020c). Guía para el diseño del producto integrador, construyendo el ensayo argumentativo. Documento inédito de trabajo.

UNESCO (1998). Conferencia Mundial sobre la Educación Superior en el siglo XXI: Visión y acción: La Educación superior en el siglo XXI, visión y acción: informe final. Francia: UNESCO. Obtenido de: https://unesdoc.unesco.org/ark:/48223/pf0000116345_spa

Umaña-Mata, A. (2020). Educación Superior en Tiempos de COVID-19: oportunidades y retos de la educación a distancia. Innovaciones Educativas, 22 (Especial):36-49. https://doi.org/10.22458/ ie.v22iEspecial.3199

VALENCIA A., O., (2021). Hacia una lectura caórdica de las políticas públicas y acciones institucionales. En Soto O., J. y Hernández F. (Coord.), Miradas de la Investigación Social y Educativa. Aportes desde la formación, las políticas y la disciplina (pp. 90-106), Xalapa, Veracruz: Universidad de Xalapa.

Valenti., G. y Flores LI. U. (2009). Ciencias sociales y políticas públicas. Revista mexicana de sociología, 71(spe):167-191. http://www.scielo.org.mx/scielo.php?script=sci_arttext\&pid=S018825032009000500007\&lng=es\&tlng=pt

Velasco, J. M., Peredo, P., Fuentes, F., González, G., y López, M. (2016). Horizonte educativo. Hacia una pedagogía de la autoorganización. México, Vereacruz: Universidad Pedagógica Veracruzana.

Villarreal, E. C. (2010). Políticas públicas. En Villareal V. H., \& Martínez, V. H. (Pre)textos para el análisis político. Disciplinas, reglas y procesos. México: Facultad Latinoamericana de Ciencias Sociales.

ZorRILLA F., M y VILLA L., L. (2003) Políticas educativas: Educación básica. Educación media superior. México: Consejo Mexicano de Investigación Educativa. 